\title{
Antioxidant and Anti-Inflammatory Effects of Blueberry Anthocyanins on High Glucose-Induced Human Retinal Capillary Endothelial Cells
}

\author{
Wuyang Huang $\mathbb{D},{ }^{1,2}$ Zheng Yan, ${ }^{1}$ Dajing Li $\mathbb{D}^{1},{ }^{1}$ Yanhong Ma, ${ }^{1}$ Jianzhong Zhou $\mathbb{D}^{1}{ }^{1}$ \\ and Zhongquan Sui $\mathbb{1}^{3}$ \\ ${ }^{1}$ Institute of Farm Product Processing, Jiangsu Academy of Agricultural Sciences, Nanjing 210014, China \\ ${ }^{2}$ Jiangsu Key Laboratory for Horticultural Crop Genetic Improvement, Jiangsu Academy of Agricultural Sciences, \\ Nanjing 210014, China \\ ${ }^{3}$ Department of Food Science and Engineering, School of Agriculture and Biology, Shanghai Jiao Tong University, \\ Shanghai 200240, China
}

Correspondence should be addressed to Jianzhong Zhou; zjzluck@126.com and Zhongquan Sui; springsui@gmail.com

Received 1 September 2017; Revised 16 November 2017; Accepted 25 December 2017; Published 22 February 2018

Academic Editor: Ilaria Peluso

Copyright ( 2018 Wuyang Huang et al. This is an open access article distributed under the Creative Commons Attribution License, which permits unrestricted use, distribution, and reproduction in any medium, provided the original work is properly cited.

Blueberries possess abundant anthocyanins, which benefit eye health. The purpose of this study was to explore the protective functional role of blueberry anthocyanin extract (BAE) and its predominant constituents, malvidin (Mv), malvidin-3-glucoside (Mv-3-glc), and malvidin-3-galactoside (Mv-3-gal), on high glucose- (HG-) induced injury in human retinal capillary endothelial cells (HRCECs). The results showed that BAE, Mv, Mv-3-glc, and Mv-3-gal enhanced cell viability $(P<0.05$ versus the HG group at $24 \mathrm{~h}$ ); decreased the reactive oxygen species (ROS, $P<0.01$ versus the HG group both at 24 and $48 \mathrm{~h}$ ); and increased the enzyme activity of catalase (CAT) and superoxide dismutase (SOD) $(P<0.05$ versus the HG group both at 24 and $48 \mathrm{~h})$. Mv could greatly inhibit HG-induced Nox4 expression both at 24 and $48 \mathrm{~h}(P<0.05)$, while BAE and Mv-3-gal downregulated Nox4 only at $48 \mathrm{~h}(P<0.05)$. Mv, Mv-3-glc, and Mv-3-gal also changed nitric oxide $(\mathrm{NO})$ levels $(P<0.05)$. $\mathrm{BAE}$ and Mv-3-glc also influenced angiogenesis by decreasing the vascular endothelial cell growth factor (VEGF) level and inhibiting Akt pathway $(P<0.05)$. Moreover, $\mathrm{Mv}$ and $\mathrm{Mv}$-3-glc inhibited HG-induced intercellular adhesion molecule-1 (ICAM-1, $P<0.001)$ and nuclear factor-kappa B $(\mathrm{NF}-\kappa \mathrm{B})(P<0.05)$. It indicated that blueberry anthocyanins protected HRCECs via antioxidant and anti-inflammatory mechanisms, which could be promising molecules for the development of nutraceuticals to prevent diabetic retinopathy.

\section{Introduction}

Diabetic retinopathy (DR) is a common complication of diabetes mellitus and a leading cause of vision loss and blindness in diabetic patients [1]. Hyperglycemia is known to be the main contributing factor in the progression of the disease, which triggers pathological metabolic and biochemical changes that damage the retinal cells [2]. In the development of DR, retinal microvascular dysfunction includes the loss of endothelial cells and pericytes, capillary occlusion and bloodretinal barrier breakdown, and endothelial cell hypertrophy and degeneration that lead to capillary nonperfusion and hypoxia $[3,4]$.

Dietary intake of phytochemicals, particularly anthocyanins, is being increasingly recognized as beneficial for modern human eye health [5]. Anthocyanins are known to have antioxidant, antimicrobial, antiviral, antiallergic, anticarcinogenic, anti-inflammatory, antimutagenic, and antiproliferative effects and thus may play an essential role in preventing various degenerative diseases including DR [6]. Anthocyanins from different vegetables and fruits, such as blueberry, bilberry, mulberry, maqui berry, blackcurrant, and black 
soybean, could reduce retinal degeneration and improve visual function [7-10]. Their possible functional mechanisms include the attenuation of oxidative damage, alteration of retinal enzyme activity, inhibition of inflammation, stimulation of the immune system, reduction of platelet aggregation, and modulation of cholesterol synthesis and hormone metabolism $[11,12]$. More and more scientists and consumers realize the vision-related benefits of anthocyanin-rich berries, and the anthocyanosides from berries are currently used in ophthalmology for their capacity to improve vision and prevent diabetic retinopathy [13].

Blueberries are rich in anthocyanins, whose content is believed to be the highest among all commonly consumed vegetables and fruits [14]. Blueberries are famous for their wide range of health benefits, including ameliorating diabetes, attenuating vascular problems, maintaining endothelial function, and preventing retinal diseases [15]. Many scientists have reported that blueberry components such as anthocyanins, polyphenols, and pterostilbene can protect corneal epithelial cells against light-induced retinal injury via antioxidative, antiangiogenic, and antiaging effects in vitro, and the bioactivity of blueberries in improving vision has been confirmed in vivo $[13,16,17]$. However, the function of blueberry anthocyanins on human retinal capillary endothelial cells (HRCECs) is still unknown. Liu et al. identified anthocyanin components of wild Chinese blueberries and found that malvidin glycosylated with hexose or pentose accounted for $>46 \%$ of the total anthocyanin content in blueberries [18]. Our previous survey also confirmed that malvidin-3glucoside (Mv-3-glc) and malvidin-3-galactoside (Mv-3-gal) were major anthocyanins in Brightwell rabbiteye blueberry (Vaccinium ashei) of Nanjing [19]. In the present study, the protective effects of blueberry anthocyanin extract, as well as malvidin and its glycosides (Mv-3-glc and $\mathrm{Mv}$-3-gal) on high glucose-induced injury in HRCECs, were investigated to propose a functional mechanism for the role of antioxidants in eye nourishment.

\section{Materials and Methods}

2.1. Materials and Chemicals. Brightwell rabbiteye blueberry (Vaccinium ashei) was harvested in July 2016 from the orchards of Lishui in Nanjing. The plant species were authenticated by a blueberry expert at the Institute of Botany, Jiangsu Province and the Chinese Academy of Sciences. The collected fruits were kept at $-18^{\circ} \mathrm{C}$ in the dark.

Human retinal capillary endothelial cells (HRCECs, primary culture cells) were purchased from Jennio Biotechnology Co. Ltd. (Guangzhou, China). Malvidin (Mv), malvidin3-glucoside (Mv-3-glc), malvidin-3-galactoside (Mv-3-gal), and trypsin were purchased from Sigma Aldrich (Shanghai, China). Fetal bovine serum (FBS) and DMEM medium were obtained from Gibco/Invitrogen (Shanghai, China). Penicillin and streptomycin were purchased from Life Technologies (Shanghai, China). Reactive oxygen species (ROS) Assay Kit, BCA (Bicinchoninic acid) Protein Assay Kit, and MTT Cell Proliferation Kit were purchased from Beyotime Institute of Biotechnology (Shanghai, China). Andygene human catalase (CAT), superoxide dismutase (SOD), and endothelial nitric oxide synthase (eNOS) Enzyme Activity Assay Kits, and nitric oxide (NO), angiotensin-converting enzyme (ACE), vascular endothelial cell growth factor (VEGF), intercellular adhesion molecule-1 (ICAM-1), and nuclear factor-kappa B (NF- $\kappa$ B) ELISA Kits were purchased from Xinzetianyou Biotechnology Co., Ltd. (Beijing, China). All chemicals and reagents were of analytical grade.

2.2. Antibodies. Rabbit monoclonal primary antibody against Nox4 was purchased from Santa Cruz Biotechnology, Inc. (Santa Cruz, CA). Rabbit polyclonal primary antibody against Akt was purchased from MengZhouShi Ruiying Biological Technology Co. Ltd. (Jiaozuo, Henan, China). Mouse monoclonal primary antibody against VEGF was purchased from Abcam (Shanghai, China). Rabbit monoclonal primary antibody against $\beta$-actin was purchased from Sigma Aldrich (Shanghai, China). Goat anti-mouse/rabbit IgG HRP-linked secondary antibodies were purchased from Cell Signaling Technology Inc. (Shanghai, China). Primary antibodies were used at $1: 1000$ dilutions. Secondary antibodies were used at $1: 4000$ dilutions.

2.3. Extraction of Anthocyanins from Blueberries. Extraction of anthocyanins from blueberries was performed according to the method used previously by Liu et al. [18]. The stored blueberry fruits were thawed at room temperature and beaten to a pulp. The blueberry pulp $(250 \mathrm{~g})$ was soaked in $1000 \mathrm{~mL}$ of methanol containing $1 \% \mathrm{HCl}$ solution for $24 \mathrm{~h}$ by mixing every two hours to extract anthocyanins. The extract was collected and centrifuged at $5000 \times \mathrm{g}$ for $15 \mathrm{~min}$. After in vacuo evaporation of the solvent at $40^{\circ} \mathrm{C}$, the residue was dissolved in $300 \mathrm{~mL}$ double distilled water and extracted with $1: 1(v / v)$ ethyl acetate three times to remove impurities, such as phenolic acids. The water phase containing anthocyanins was collected and concentrated in vacuo to obtain the crude anthocyanin extract. The extract was further purified with AB-8 macroporous resin (Sigma Aldrich, China). The extract was subjected to column chromatography on $1000 \mathrm{~g}$ AB-8 macroporous resin for $24 \mathrm{~h}$ absorption and then eluted with double distilled water to remove fructose and protein. Anthocyanin fraction was eluted with $80 \%$ ethanol containing $1 \% \mathrm{HCl}$ solution, concentrated in vacuo, and then dried using an Eyela FDU-1200 freeze dryer (Tokyo Rikakikai, Japan) to get blueberry anthocyanin extract (BAE) powder $(0.3 \mathrm{~g})$. Blueberry anthocyanin extract was characterized by HPLC-DAD to determine the exact amount of each compound in the extract. Malvidin glycosides were the dominant anthocyanins in the blueberries, accounting for $47.9 \%$ of total anthocyanin content, in which $\mathrm{Mv}$-3-glc and $\mathrm{Mv}$-3-gal were $17.2 \%$ and $22.6 \%$, respectively (unpublished data).

\subsection{Endothelial Cell Culture and Treatment. HRCECs were} cultured in normal glucose $(5.5 \mathrm{mmol} / \mathrm{L})$ DMEM containing $10 \% \mathrm{FBS}$ and $1 \%$ streptomycin and penicillin at $37^{\circ} \mathrm{C}$ in a $5 \%$ $\mathrm{CO}_{2}$ humidified incubator. The third to fifth passage cells were used for all experiments at $80-90 \%$ confluence. HRCECs were quiesced in a reduced serum medium (not containing FBS) for $4 \mathrm{~h}$ before the experiment. Based on the preliminary experiments, a dose-response assay regarding 
viability showed that the extract and compounds were not toxic to the cells at a concentration of less than $10 \mu \mathrm{g} / \mathrm{mL}$. The cells were seeded in 6-well plates and pretreated with $10 \mu \mathrm{g} / \mathrm{mL}$ of BAE, Mv, Mv-3-glc, or Mv-3-gal for $24 \mathrm{~h}$, respectively. Then, the sample cells were stimulated with high glucose (final concentration $30 \mathrm{mmol} / \mathrm{L}$ ) for another 24 and $48 \mathrm{~h}$. Normal glucose (NG: $5.5 \mathrm{mmol} / \mathrm{L}$ ) group without pretreating blueberry anthocyanins was used as the control. High glucose (HG: $30 \mathrm{mmol} / \mathrm{L}$ ) group without pretreating blueberry anthocyanins was used as the oxidative model. The supernatants were collected for ELISA analysis. The cells were prepared for Western blotting.

2.5. Cell Viability Detection. The cell viability was determined by MTT method. The cells were cultured with high glucose $(30 \mathrm{mmol} / \mathrm{L}$ ) for 24 and $48 \mathrm{~h}$ with or without BAE, Mv, Mvglc, or Mv-gal pretreatment for $24 \mathrm{~h}$. Ten microliters of MTT $(5 \mathrm{mg} / \mathrm{mL})$ was added to each well and cultured for $4 \mathrm{~h}$. After the removal of MTT solution, cell crystal was dissolved by adding $100 \mu \mathrm{L}$ DMSO (dimethylsulfoxide) and shaking $10 \mathrm{~min}$ slowly. The absorbance was measured at $550 \mathrm{~nm}$ on a Synergy H4 Multi-Mode Microplate Reader (BioTek Instruments, Inc., Winooski, VT, USA). The reader was controlled via Hyper Terminal Applet ELISA software. The cells were cultured only when normal glucose $(5.5 \mathrm{mmol} / \mathrm{L})$ was used as the control group. The blank group used the wells without cells. The cell viability was determined with the following formula: Cell viability $(\%)=($ sample group OD value - blank group OD value)/(control group OD value - blank group OD value $) \times 100 \%$.

2.6. Reactive Oxygen Species (ROS) Assay. Dichloro-dihydrofluorescein diacetate (DCFH-DA) detection kit was used to assess the ROS level in HRCECs. Briefly, the cells were seeded in 96-well plates, treated with different samples to incubate for $24 \mathrm{~h}$ and cultured with high glucose $(30 \mathrm{mmol} / \mathrm{L})$ for 24 and $48 \mathrm{~h}$. After washing cells with phosphate-buffered saline (PBS), $10 \mu \mathrm{mol} / \mathrm{L}$ DCFH-DA was added to each well and reacted for $20 \mathrm{~min}$ at $37^{\circ} \mathrm{C}$. The cells were collected after dissociation, and fluorescence was recorded by a Synergy H4 Multi-Mode Microplate Reader (BioTek Instruments, Inc., Winooski, VT, USA) with 488-P excitation and 525-P emission filters. The total fluorescence intensity of cells in each well was noted, and ROS generation was measured as fold of the control (each NG group, 24 and $48 \mathrm{~h}$ ).

2.7. ELISA Analysis and Western Blotting. The levels of CAT, SOD, NO, eNOS, ACE, VEGF, ICAM-1, and NF- $\kappa$ B were quantified from the supernatants using ELISA Kits or enzyme activity assay kits. The assay procedure was employed according to the kit protocol booklet instructions. The absorbance was measured at $450 \mathrm{~nm}$ on a Synergy H4 Multi-Mode Microplate Reader (BioTek Instruments, Inc. Winooski, VT, USA). The reader was controlled via Hyper Terminal Applet ELISA software. The total cell protein of the supernatant in each well was detected using BCA Protein Assay Kit.

Protein expression of Nox4, Akt, and VEGF was also analyzed by Western blotting performed on the HRCEC lysates. Data were normalized by reprobing the membrane with an antibody against $\beta$-actin, which was used as a loading control. Cell lysates from untreated cells were loaded on to every gel, and all data were expressed as fold of the corresponding control.

2.8. Statistical Analysis. All data presented are mean value \pm standard deviation (SD) of three independent experiments. Figures were obtained using GraphPad Prism Version 5 (GraphPad Software, Inc., CA, USA). The data of each group used $t$-tests were to determine significant differences among different treatments (NG, HG, BAE, Mv, Mv-3-glc, and Mv-3-gal). Two-way analysis of variance (ANOVA) was used to analyze differences in culture time (24 and $48 \mathrm{~h})$ and their interactions with treatment. The differences were considered significant at $P$ value $<0.05$.

\section{Results}

3.1. Effects of Blueberry Anthocyanins on Cell Viability. In this study, we observed that stimulation with high glucose concentration $(30 \mathrm{mmol} / \mathrm{L}, \mathrm{HG}$ : $64.03 \pm 2.97 \%)$ for $24 \mathrm{~h}$ significantly decreased cell viability in comparison with normal glucose- $(5.5 \mathrm{mmol} / \mathrm{L}, \mathrm{NG}: 100 \pm 7.04 \%)$ incubated cells $(P<0.01)$. MTT assay showed that $10 \mu \mathrm{g} / \mathrm{mL}$ of BAE, Mv, $\mathrm{Mv}-3$-glc, and Mv-3-gal all significantly increased cell viability after $24 \mathrm{~h}$ of high glucose incubation $(P<0.05)$ (Figure 1$)$. The effect of blueberry anthocyanin extract (BAE: 78.69 \pm $4.75 \%$ ) was lower than those of malvidin and its glycosides, while the effects of malvidin glycosides were stronger than that of malvidin (Mv: $82.78 \pm 10.27 \%$ ). Malvidin-3glucoside (Mv-3-glc: $91.16 \pm 7.77 \%)$ was better than malvidin-3-galactoside (Mv-3-gal: $86.49 \pm 3.32 \%)$. These indicated that high glucose inhibited cell growth, while $\mathrm{BAE}$, malvidin, and its glycosides protected the cell by inhibiting a decrease in cell viability caused by HG. Interestingly, the same effects were not found in cells incubated with HG for a long time (significant difference between 24 and $48 \mathrm{~h}: P<0.01$ ). The cells continued to grow over time with more cell numbers at $48 \mathrm{~h}$ than at $24 \mathrm{~h}$ in each well. However, no significant difference was observed between NG $(100 \pm 5.28 \%)$ and HG $(106.01 \pm 10.41 \%)$, while anthocyanin pretreatments seemed to decrease a little cell viability (BAE: $82.55 \pm 8.90 \%, \mathrm{Mv}$ : $94.42 \pm 10.49 \%, \mathrm{Mv}-3$-glc: $89.76 \pm 10.01 \%$, and Mv-3-gal: $77.84 \pm 5.57 \%)$.

3.2. Effects of Blueberry Anthocyanins on ROS, CAT, SOD, and Nox4 Levels. As shown in Figure 2, high glucose concentration significantly enhanced the ROS levels of HRCECs at both short-term and a long-term $(P<0.001)$. ROS levels of the HG groups at 24 and $48 \mathrm{~h}$ were $4.23 \pm 0.41-$ and $4.53 \pm$ 0.23 -folds of the control (NG groups), respectively. BAE, $\mathrm{Mv}, \mathrm{Mv}-3$-glc, and $\mathrm{Mv}$-3-gal all significantly inhibited HG-induced increase of ROS in HRCECs $(P<0.01$ for $24 \mathrm{~h} ; P<0.001$ for $48 \mathrm{~h})$. HRCECs exhibited higher ROS levels at $48 \mathrm{~h}$ than those at $24 \mathrm{~h}(P<0.001)$ except pretreatment with Mv-3-glc. Similarly, malvidin-3-glucoside possessed the strongest antioxidant effect, with nearly the same ROS levels as the control. Moreover, the ROS level of cells treated with $\mathrm{Mv}$-3-glc was only $0.93 \pm 0.23$-fold of the control 


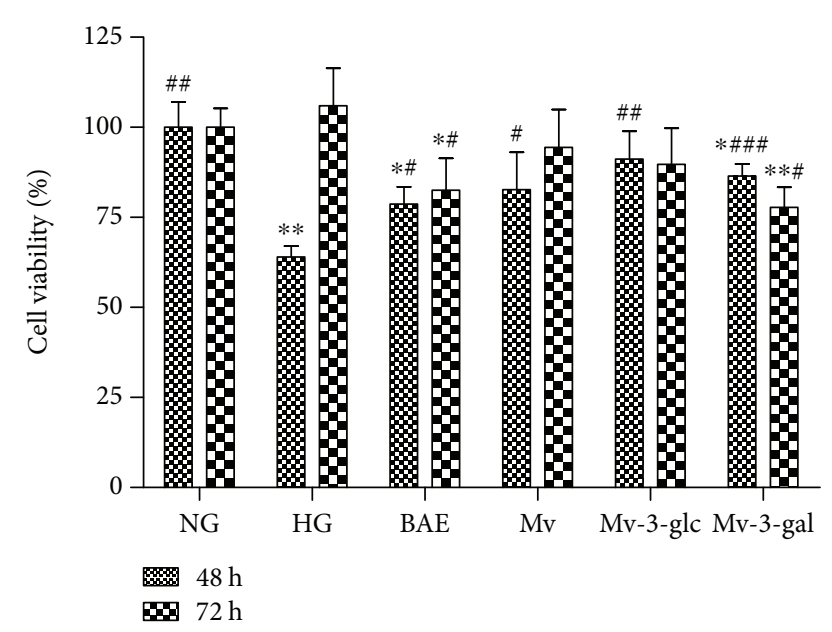

FIGURE 1: Effects of different treatment on cell viability in HRCECs exposed to high glucose for 24 and $48 \mathrm{~h}$. * and $* *$ indicate $P<0.05$

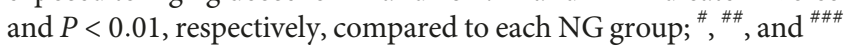
indicate $P<0.05, P<0.01$, and $P<0.001$, respectively, compared to each $\mathrm{HG}$ group.

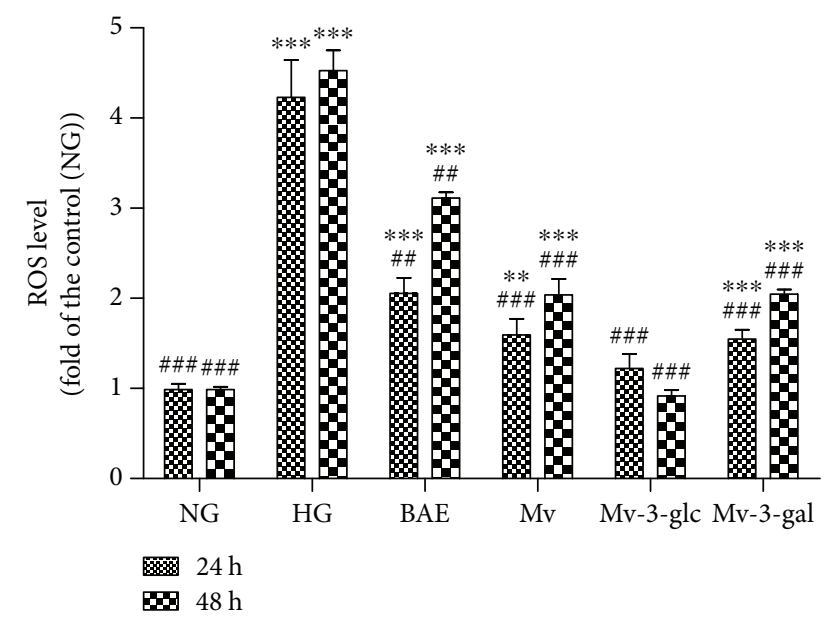

Figure 2: Effects of different treatment on ROS levels in HRCECs exposed to high glucose for 24 and $48 \mathrm{~h} . * *$ and $* * *$ indicate $P<0.01$ and $P<0.001$, respectively, compared to each NG group; $\#$ and ${ }^{\# \# \#}$ indicate $P<0.01$ and $P<0.001$, respectively, compared to each HG group.

when exposed to high glucose for $48 \mathrm{~h}$. These data suggest that BAE, malvidin, and their glycosides significantly reduced the levels of ROS, showing an effective attenuation of high glucose-induced oxidative damage in HRCECs.

In this study, high glucose reduced the activity of the antioxidant enzymes CAT $(P<0.01$ at $24 \mathrm{~h})$ and SOD $(P<0.05$ at $48 \mathrm{~h}$ ) in HRCEC supernatants (Figure 3). BAE, Mv, Mv-3-glc, and $\mathrm{Mv}$-3-gal all significantly enhanced CAT and SOD activity in HRCEC supernatants $(P<0.05)$. The CAT and SOD enzyme activity of cells pretreated with BAE, $\mathrm{Mv}, \mathrm{Mv}-3$-glc, and Mv-3-gal was even higher than that of the control (NG group). BAE and $\mathrm{Mv}$-3-gal seemed to have more pronounced effects on SOD activity than Mv and Mv-3-glc. However, $\mathrm{Mv}$ and Mv-3-glc seemed to have more pronounced effects on CAT activity than BAE and Mv-3-gal. Both CAT and
SOD enzyme activity decreased in a time-dependent manner $(24 \mathrm{~h}$ versus $48 \mathrm{~h}: P<0.01)$. Thus, blueberry anthocyanin malvidin and its glycosides could attenuate oxidative stress by upregulating the antioxidant enzymes activities in HRCECs.

Figure 4(a) showed that high glucose significantly enhanced Nox 4 protein expression in cells to 2.17 -fold of the control after $24 \mathrm{~h}$ stimulation $(P<0.01)$, but it had no significant change for $48 \mathrm{~h}$ stimulation. Only $\mathrm{Mv}$ could greatly inhibit Nox 4 expression induced by high glucose stimulation for $24 \mathrm{~h}$ with inhibition rate at $45.96 \%(P<0.05)$. BAE, Mv, and Mv-3-gal all could lower Nox4 levels in cells with $48 \mathrm{~h}$ HG stimulation $(P<0.05$ for BAE and Mv-3-gal; $P<0.01$ for Mv). However, Mv-3-glc did not affect the Nox4 protein expression in cells at both 24 and 48 hours. Nox 4 protein expression also decreased in a time-dependent manner $(24 \mathrm{~h}$ versus $48 \mathrm{~h}: P<0.01)$. The results indicated that $\mathrm{Mv}$ could reduce superoxide production in endothelial cells by downregulating Nox4. Mv-3-glc inhibited the most ROS formation but did not inhibit Nox4 expression, which should exist some other mechanism for Mv-3-glc on antioxidant effects in HRCECs.

3.3. Effects of Blueberry Anthocyanins on NO, eNOS, and ACE Levels. In this study, high glucose could significantly increase the NO level $(P<0.001$ at $24 \mathrm{~h} ; P<0.01$ at $48 \mathrm{~h})$ and eNOS activity $(P<0.01$ at $24 \mathrm{~h} ; P<0.05$ at $48 \mathrm{~h})$ in HRCEC supernatants (Figures 5(a) and 5(b)). BAE slightly inhibited the increase of $\mathrm{NO}$ at $24 \mathrm{~h}(P>0.05)$ but not as much as Mv-3glc and $\mathrm{Mv}$-3-gal $(P<0.001)$. However, $\mathrm{Mv}$-3-glc and $\mathrm{Mv}$ 3 -gal seemed to induce more NO than the HG group for long time incubation $(P<0.01$ for $\mathrm{Mv}$-3-glc and $P<0.001$ for Mv-3-gal at $48 \mathrm{~h}$ ). HRCECs produced higher $\mathrm{NO}$ at $24 \mathrm{~h}$ than those at $48 \mathrm{~h}$. Enzyme activity of eNOS in HRCECs pretreated with $\mathrm{BAE}, \mathrm{Mv}, \mathrm{Mv}$-3-glc, and $\mathrm{Mv}$-3-gal was higher than that of NG and lower than that of HG. However, the differences among them were not always significant. Like CAT and SOD, eNOS enzyme activity decreased in a timedependent manner $(24 \mathrm{~h}$ versus $48 \mathrm{~h}: P<0.001)$. The change of ACE content in the supernatants showed that high glucose-induced ACE expression $(P<0.01$ at $24 \mathrm{~h})$. Malvidin downregulated the HG-induced ACE expression, while BAE, $\mathrm{Mv}$-3-glc, and $\mathrm{Mv}$-3-gal further upregulated ACE expression (Figure 5(c)). ACE contents also decreased in a timedependent manner $(24 \mathrm{~h}$ versus $48 \mathrm{~h}: P<0.001)$.

3.4. Effects of Blueberry Anthocyanins on High GlucoseInduced VEGF and Akt Levels. Figure 6 shows that high glucose concentration could significantly upregulate the VEGF production in HRCEC supernatants $(P<0.01$ at $24 \mathrm{~h} ; P<0.001$ at $48 \mathrm{~h}$ ). BAE, Mv, Mv-3-glc, and Mv-3-gal significantly inhibited HG-induced VEGF production at $24 \mathrm{~h}(P<0.01$ for BAE, Mv, and Mv-3-glc; $P<0.05$ for Mv3 -gal). Mv and Mv-3-glc also exhibited inhibitory effects at $48 \mathrm{~h}(P<0.01)$. However, BAE and $\mathrm{Mv}-3$-gal did not show the VEGF inhibitory effect at $48 \mathrm{~h}$. Like the contents of NO and ACE and the enzyme activity of CAT, SOD, and eNOS, VEGF contents were decreased with a prolonged time $(24 \mathrm{~h}$ 


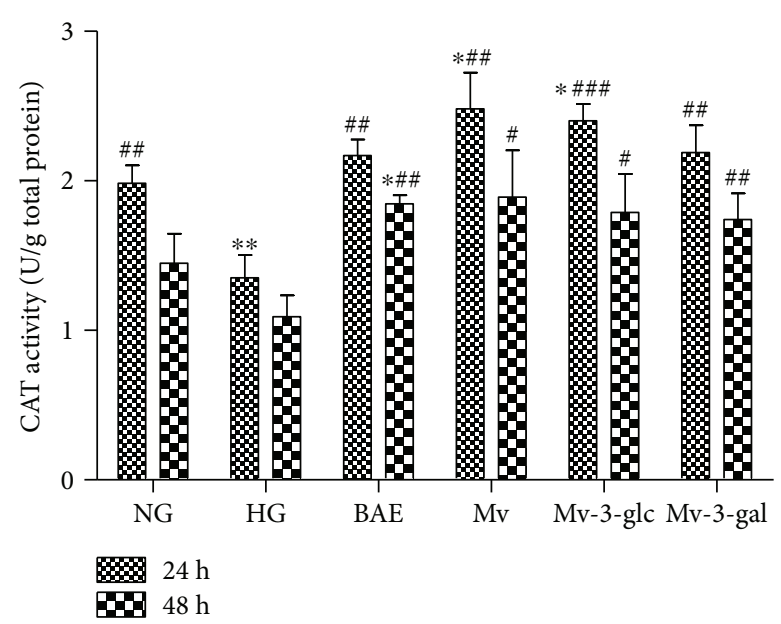

(a)

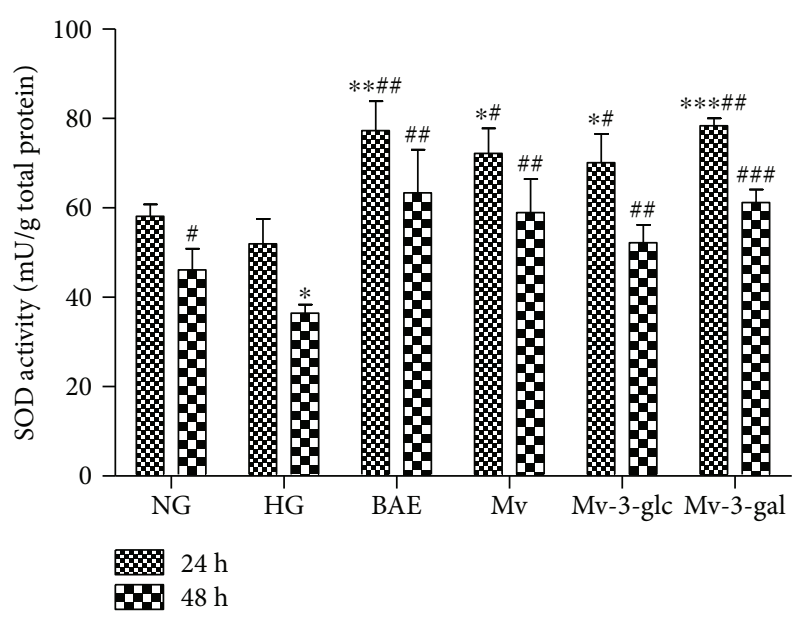

(b)

FIGURE 3: Effects of different treatment on CAT (a) and SOD activity (b) in HRCECs exposed to high glucose for 24 and 48 h. *, **, and *** indicate $P<0.05, P<0.01$, and $P<0.001$, respectively, compared to each NG group; ${ }^{\#}$ \#\#, and ${ }^{\# \# ~}$ indicate $P<0.05, P<0.01$, and $P<0.001$, respectively, compared to each $\mathrm{HG}$ group.

versus $48 \mathrm{~h}: P<0.001)$. Mv-3-glc showed the strongest inhibitory effects against VEGF.

VEGF protein expression in cells was also determined by Western blotting (Figure 4(b)). Strangely, high glucose did not enhance VEGF protein expression in cells and even $24 \mathrm{~h}$ HG stimulation decreased VEGF level to 0.64-fold of the control $(P<0.05)$. BAE could greatly inhibit VEGF expression both at $24 \mathrm{~h}(0.59$-fold of the control) and $48 \mathrm{~h}$ (0.73-fold) $(P<0.001$ and $P<0.01$ versus the control). Moreover, Mv-3-glc exhibited lower VEGF protein level $(0.69$-fold $)$ than the control $(P<0.05)$. VEGF protein expression in cells at 24 and $48 \mathrm{~h}$ was different $(P<0.05)$. The VEGF levels at $48 \mathrm{~h}$ pretreated with BAE and Mv were higher than those at $24 \mathrm{~h}$, which was consistent with the HGstimulated group. However, the VEGF levels at $48 \mathrm{~h}$ pretreated with Mv-3-glc and Mv-3-gal were lower than those at $24 \mathrm{~h}$, which indicated their capacity to downregulate the VEGF expression.

Moreover, high glucose could enhance the expression of Akt protein in cells to $1.18-(P<0.01)$ and $1.11-(P<0.05)$ folds of the control after 24 and $48 \mathrm{~h}$ stimulation, respectively. BAE, Mv-3-glc, and Mv-3-gal inhibited the increased Akt expression with HG stimulation for $24 \mathrm{~h}$ or $48 \mathrm{~h}$. The Akt protein levels of BAE, Mv-3-glc, and Mv-3-gal at $24 \mathrm{~h}$ were 0.76-, 0.91-, and 1.09-folds of the control, respectively, and at $48 \mathrm{~h}$, they were 1.06-, 1.01-, 0.89-folds of the control, respectively (Figure 4(c)). However, $\mathrm{Mv}$ did not affect the Akt protein expression in cells.

3.5. Effects of Blueberry Anthocyanins on High GlucoseInduced ICAM-1 and NF- $\kappa B$. In this study, high glucose concentrations could significantly upregulate the ICAM-1 production in HRCEC supernatants $(P<0.001$ both at 24 and $48 \mathrm{~h})$. As shown in Figure 7(a), malvidin and $\mathrm{Mv}-3$-glc greatly inhibited HG-induced ICAM- 1 levels $(P<0.001$ both at 24 and $48 \mathrm{~h}$ ), particularly $\mathrm{Mv}$ exhibited a lower ICAM-1 content than NG at $24 \mathrm{~h}(P<0.01)$. Mv-3-gal was also observed to have an ICAM-1 inhibitory effect compared to the HG group $(P<0.01$ at $24 \mathrm{~h}$ and $P<0.05$ at $48 \mathrm{~h})$. However, BAE had no effects on ICAM-1 levels in HRCECs at $24 \mathrm{~h}$. ELISA assay of NF- $\kappa \mathrm{B}$ (p65) contents showed similar results. However, the effects were not always significant (Figure 7(b)). Moreover, $\mathrm{Mv}$ and $\mathrm{Mv}$-3-glc exhibited strongest inhibitory effects at both 24 and $48 \mathrm{~h}(P<0.05)$. The ICAM- 1 contents and NF- $\kappa$ B (p65) levels of $48 \mathrm{~h}$ were lower than those of $24 \mathrm{~h}(P<0.001)$ except $\mathrm{Mv}$, which had a lower ICAM- 1 content at $24 \mathrm{~h}$ than that at $48 \mathrm{~h}(P<0.01)$.

\section{Discussion}

The chronic hyperglycemia-induced cell death in the endothelial cells of retinal vessels is well established [2]. Leal et al. found that high glucose, but not mannitol, decreased cell viability of rat retinal endothelial cells exposed to $30 \mathrm{mM}$ glucose (high glucose) for 7 days (long-term exposure) [20]. Fan et al. also observed that stimulation with $30 \mathrm{mM}$ glucose for $48 \mathrm{~h}$ and $72 \mathrm{~h}$ significantly decreased cell viability in comparison with $5.5 \mathrm{mM}$ glucose-treated rat retinal capillary endothelial cells [1]. However, Wang et al. reported that high glucose-induced human retinal microvascular endothelial cell proliferation and enhanced the cell viability [2]. In the present study, cell viability of human retinal capillary endothelial cells was decreased by high glucose only when stimulated for $24 \mathrm{~h}$ but not for $48 \mathrm{~h}$. Maybe the effect of cell proliferation counteracted the effect of cell death at $48 \mathrm{~h}$, so there was no significant change in cell viability. Moreover, blueberry anthocyanin extract and its major constituents (malvidin glycosides) could have a particular protective effect on HRCECs, all enhancing cell viability. Their possible functional mechanisms include attenuation of oxidative damage, alteration of retinal enzyme activity, and inhibition of inflammation [6]. As a whole extract, BAE exhibited a reductionist activity, since crude extract also had some other noneffective components. This confirmed that anthocyanins, particularly 


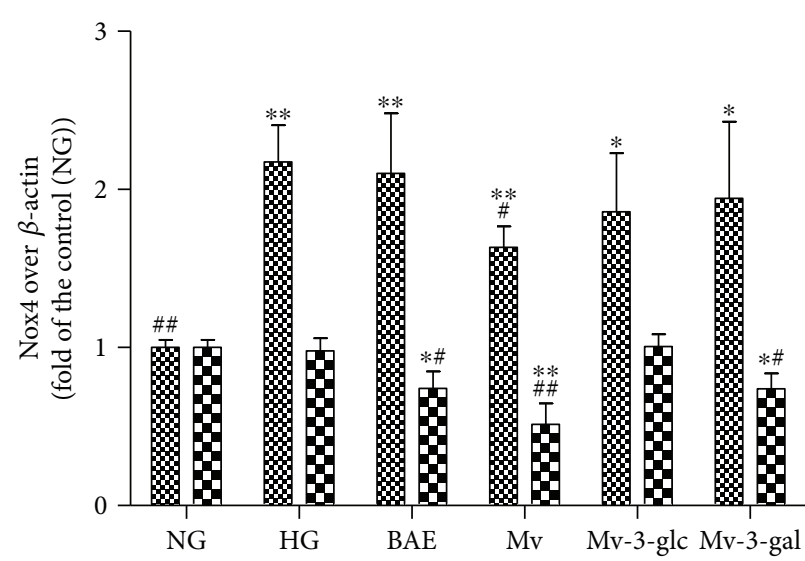

\% $24 \mathrm{~h}$ I8 48

(a)

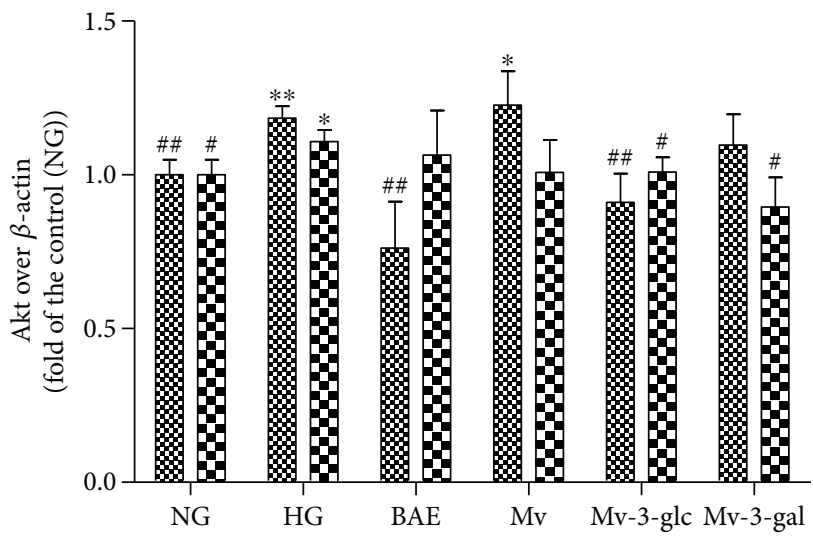

$\% 24 \mathrm{~h}$

D8 48

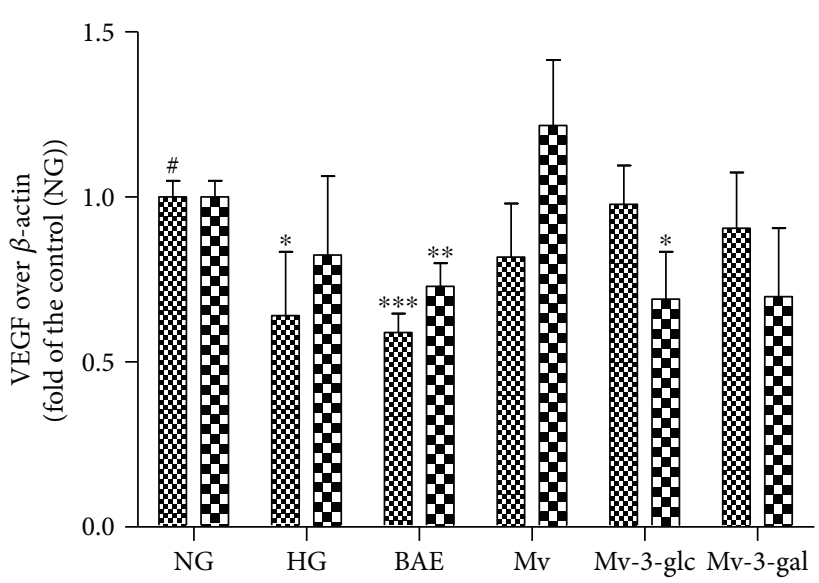

8) $24 \mathrm{~h}$

$48 \mathrm{~h}$

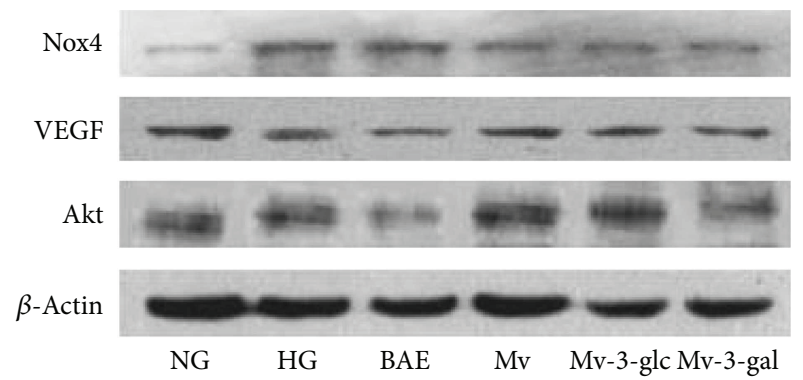

(d)

Figure 4: Effects of different treatment on Nox4 (a), VEGF (b), and Akt (c) protein expression in HRCECs exposed to high glucose for 24 and 48 h. (d) Representative Western blots of HRCECs exposed to high glucose for $24 \mathrm{~h}$ are shown. ${ }^{*},{ }^{* *}$, and ${ }^{* * *}$ indicate $P<0.05, P<0.01$, and $P<0.001$, respectively, compared to each NG group; ${ }^{\#}$ and ${ }^{\# \#}$ indicate $P<0.05$ and $P<0.01$, respectively, compared to each $\mathrm{HG}$ group.

Mv-3-gal and Mv-3-glc, were effective components in blueberry extracts.

The upregulated reactive oxygen species (ROS) generation inferred the potent stimuli for the oxidative stress [21]. In this study, BAE, Mv, Mv-3-glc, and Mv-3-gal attenuated high glucose-induced oxidative stress in HRECE by reducing ROS levels. The activity of nicotinamide adenine dinucleotide phosphate (NADPH) oxidases has been identified as an important source of ROS in vascular endothelial cells [22]. Nox4 is the major catalytic component of an endothelial NADPH oxidase. The self-perpetuating cycle between oxidative stress and inflammation contributes to the upregulation of NADPH oxidase, which increases ROS production [23]. The ROS can react with nitric oxide (NO) to form peroxynitrate leading to a reduction in $\mathrm{NO}$ bioavailability and subsequently impaired NO-dependent vasodilation [15]. However, Schröder et al. speculated that endogenous Nox4 protects the vasculature against inflammatory stress because loss of Nox4 results in the reduction of endothelial nitric oxide synthase (eNOS) and heme oxygenase-1 (HO-1) expression, as well as NO production, which comprise an important antioxidant defense against endothelial oxidative damage [23]. This might explain why Nox4 did not change greatly with pretreatment with blueberry anthocyanin malvidin glycosides in this study.

The local production of NO mediates the function of endothelium-dependent vasodilation, which is synthesized from $L$-arginine by the enzyme eNOS [24]. Conversely, angiotensin-converting enzyme (ACE) is responsible for vasoconstriction by converting angiotensin I (AngI) into AngII (a potent vasoconstrictor) and inactivating the vasodilator bradykinin [25]. Vasodilation and vasoconstriction of blood vessels together affect blood pressure. In several pathological conditions including diabetes, endotheliumdependent vasodilation is reduced because of a decreased release of NO [15, 26]. Extracts from various plants full of anthocyanins can induce endothelium-dependent vasodilation probably by releasing NO or enhancing NO bioactivity 

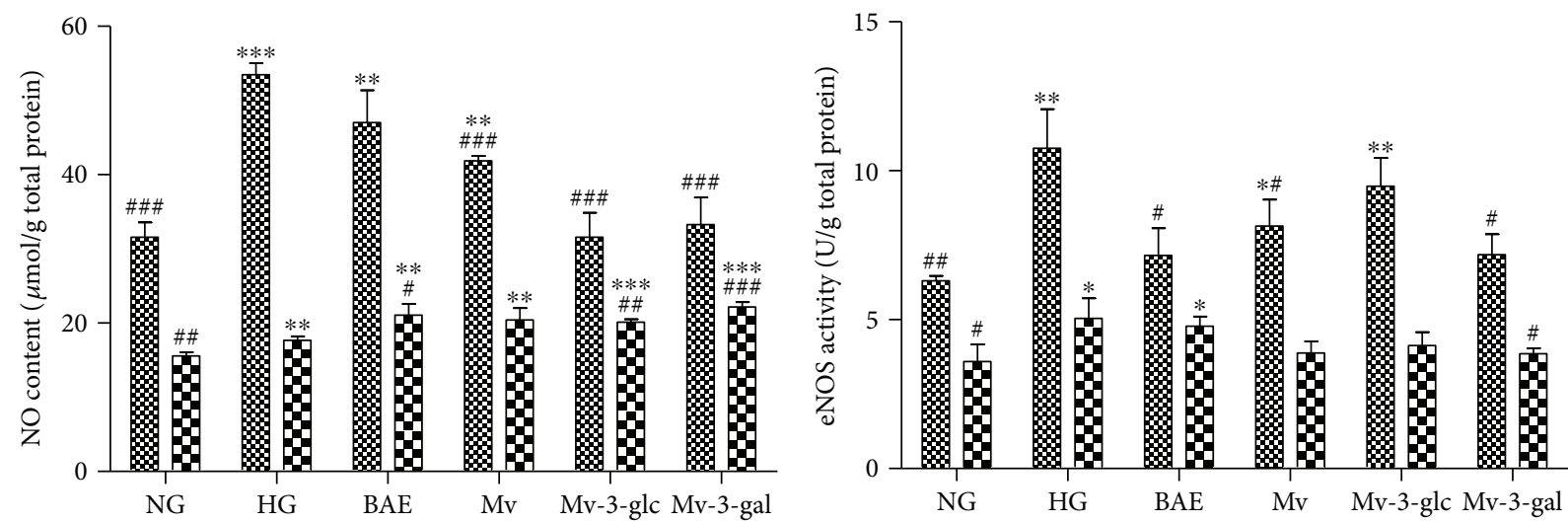

8.8. $24 \mathrm{~h}$

$24 \mathrm{~h}$

Q $48 \mathrm{~h}$

(a)

(b)

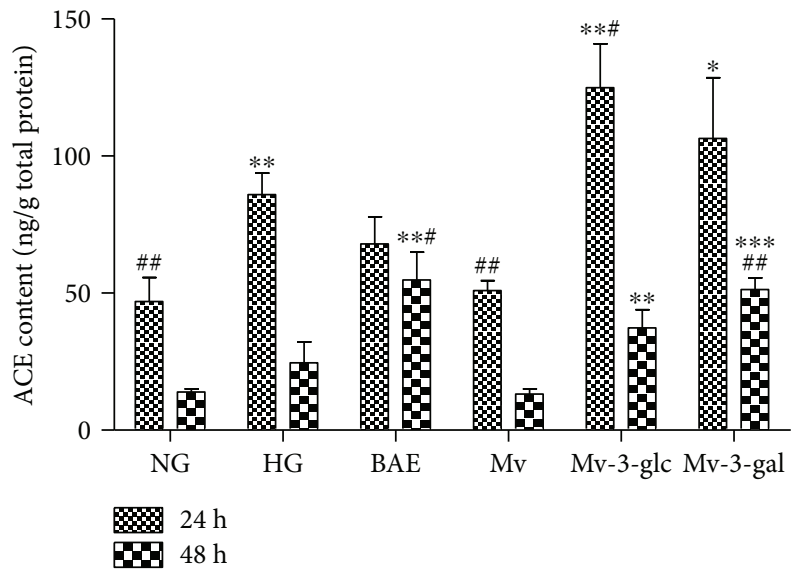

(c)

FIGURE 5: Effects of different treatment on NO contents (a), eNOS activity (b), and ACE contents (c) in HRCECs exposed to high glucose for 24 and 48 h. $^{*},{ }^{* *}$, and ${ }^{* * *}$ indicate $P<0.05, P<0.01$, and $P<0.001$, respectively, compared to each NG group; ${ }^{\#}{ }^{\# \#}$, and ${ }^{\# \#}$ indicate $P<0.05$, $P<0.01$, and $P<0.001$, respectively, compared to each HG group.

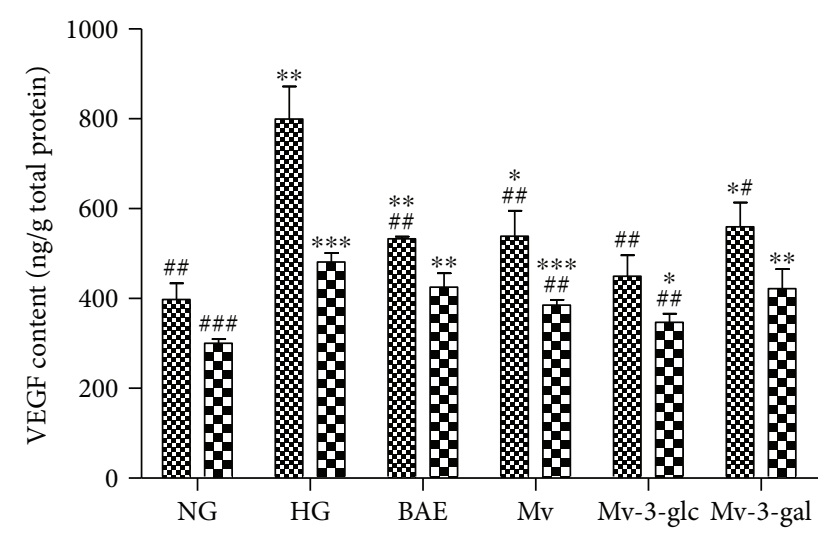

$824 \mathrm{~h}$

II $48 \mathrm{~h}$

Figure 6: Effects of different treatment on VEGF contents in HRCECs exposed to high glucose for 24 and $48 \mathrm{~h} .{ }^{*},{ }^{* *}$, and *** indicate $P<0.05, \quad P<0.01$, and $P<0.001$, respectively, compared to each NG group; \#, \#\#, and \#\# indicate $P<0.05$, $P<0.01$, and $P<0.001$, respectively, compared to each HG group.
[27]. For example, blackcurrant prevented eye probably by increasing blood supply based on the endothelium vasodilation [28]. Strangely, the effects on NO production were not always consistent with the effects on eNOS activity in this study. The level of NO could not be completely determined by eNOS because endothelial NOS is only one of the three isoforms of nitric oxide synthase [29]. Other isoforms, such as neuronal NOS (nNOS) and inducible NOS (iNOS), should also be effective. Retinal capillary endothelial cells can express iNOS when stimulated by inflammatory mediators, such as IL-1 $\beta$ [21]. Moreover, nitrosative/oxidative stress might be related to NO levels. NOS can also catalyze superoxide anion production, depending on substrate and cofactor availability [29]. Anthocyanins exhibited high NO levels, which contributed to the effective antioxidant capacity and the vasodilatory effect. Our previous study found that malvidin and its glycosides could inhibit TNF- $\alpha$-induced ACE expression and activity in the human umbilical vein endothelial cells [30]. However, blueberry anthocyanins did not downregulate ACE expression and were not detected in HRCECs in the present study. It seemed to be contradictory 


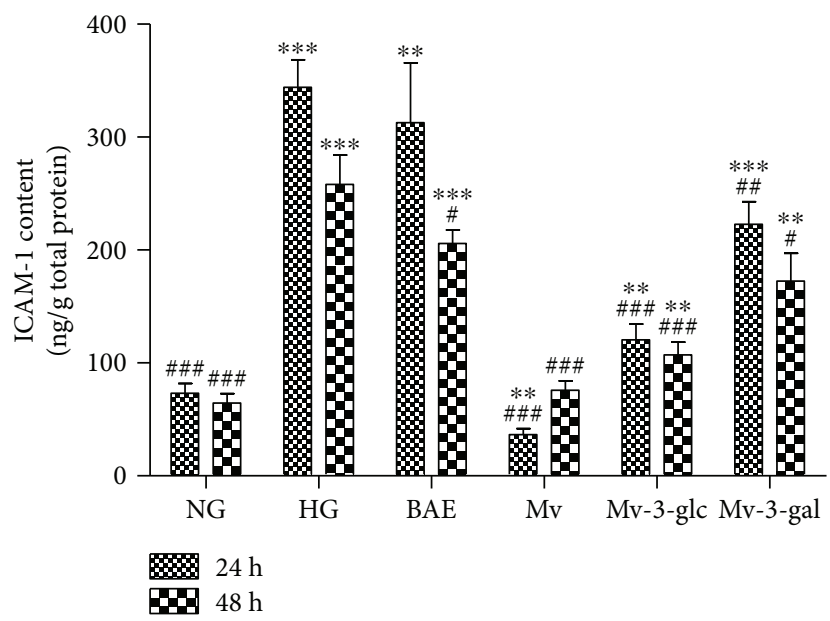

(a)

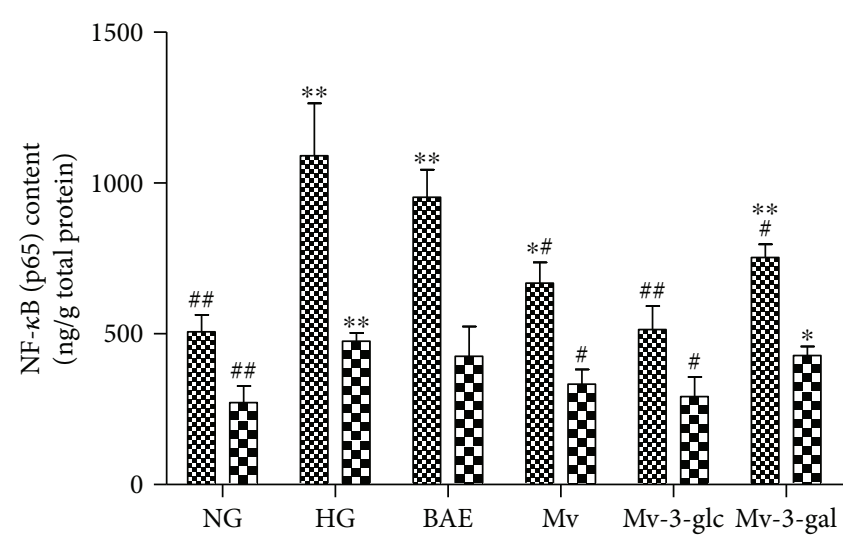

\& $24 \mathrm{~h}$ EDh

FIGURE 7: Effects of different treatment on ICAM (a) and NF- $\kappa$ B (p65) contents (b) in HRCECs exposed to high glucose for 24 and 48 h. *, ${ }^{* *}$, and ${ }^{* * *}$ indicate $P<0.05, P<0.01$, and $P<0.001$, respectively, compared to each NG group; ${ }^{\#}$, , and ${ }^{\# \#}$ indicate $P<0.05, P<0.01$, and $P<0.001$, respectively, compared to each HG group.

that BAE and malvidin glycosides possessed simultaneous vasodilatory and vasoconstrictive effects at the same time. Clozel et al. also reported that the endothelin ETB receptor could mediate both vasodilation and vasoconstriction [31]. Further study on the mechanism of malvidin glycosides in the endothelium should be conducted in the future.

Under retinopathy state, the activity of NOS is spontaneously regulated to improve the formation of NO fighting for inflammation [29]. Moreover, the stimulus-secretion coupling of high glucose-induced the synthesis and the release of NO could interact with the vascular endothelial growth factor (VEGF) [32]. VEGF stimulates vasculogenesis and angiogenesis, which gives rise to the proliferative DR [33]. In the diabetic microangiopathic condition, microvascular permeability and the number of leucocytes sticking to the vascular endothelium are increased [6]. In the present study, high glucose stimulated VEGF secretion, and blueberry anthocyanin malvidin glycosides downregulated the VEGF expression.

Akt, the serine/threonine kinase, is a central node in cell signaling downstream responses, including cell survival, growth, proliferation, angiogenesis, glucose uptake, metabolism, and migration [34]. In endothelial cells, the PI3K-Akt pathway is robustly activated by VEGF, and Akt activates eNOS [35]. The release of NO produced by activated eNOS can stimulate vasodilation, vascular remodeling, and angiogenesis [36]. During diabetic retinopathy, Akt activation is aberrant. In the present study, blueberry anthocyanin malvidin glycosides inhibited Akt expression, therefore inhibiting eNOS activity and changing the NO level.

Nuclear factor-kappa B (NF- $\kappa \mathrm{B})$, an important protein complex that controls the transcription of DNA and cytokine production, is present in many cell types and participates in cell apoptosis and neovascularization [37]. On activation of the NF- $\kappa \mathrm{B}$ pathway, the $\mathrm{p} 65$ protein interacts with the promoters to induce and maintain the state of inflammation [38]. It has been shown that the expression of NF- $\kappa B$ p 65 in the retina can regulate the expression of intercellular adhesion molecule-1 (ICAM-1), which is the primary adhesion molecule responsible for inflammation in the pathogenesis of diabetic retinopathy [39]. Previous studies confirmed that $\mathrm{Mv}$-3-glc could increase NO bioavailability as well as inhibit peroxynitrite-induced NF- $\kappa$ B activation [40]. Anthocyanin and phenolic acid metabolites were found to attenuate visible light-induced retinal degeneration in vivo via NF- $\kappa \mathrm{B}$ suppression [41]. In the present study, blueberry anthocyanin malvidin glycosides also exhibited anti-inflammatory effects by inhibiting NF- $\kappa \mathrm{B}$ in HRCECs.

\section{Conclusions}

In the present study, blueberry anthocyanin extract, as well as its major constituent malvidin and its glycosides, could protect human retinal capillary endothelial cells against high glucose-induced injury. BAE, Mv, Mv-3-glc, and Mv-3-gal promoted cell growth of HRCECs with higher cell viability than the high glucose-stimulated group. They had great antioxidant effects by decreasing ROS levels and increasing enzyme activity of CAT and SOD in HRCECs. Downregulation of Nox4 expression might be an antioxidant mechanism. Moreover, the upregulation of NO levels might be another antioxidant mechanism for blueberry anthocyanins, which contributed to vasodilatory effects. However, malvidin glycosides still possessed vasoconstrictory effects by increasing ACE contents in some case. Blueberry anthocyanins and malvidin glycosides changed VEGF levels in HRCECs and influenced the Akt pathway to some extent. Moreover, Mv and $\mathrm{Mv}$-3-glc significantly inhibited HG-induced extracellular ICAM- 1 and NF- $\kappa$ B (p65). BAE, Mv, Mv-3-glc, and Mv3 -gal protected cells in a time-dependent manner with the difference between 24 and $48 \mathrm{~h}$ HG stimulation. Incubation for long times may weaken the protective effects, which can be attributed to the irreparable oxidative damage caused by the prolonged stimulation of high glucose. The results 
indicated that blueberries, as an excellent resource of anthocyanins, could improve human retinal capillary endothelial function and, thereby, might have the potential to prevent the progression of diabetic retinopathy.

\section{Conflicts of Interest}

There are no conflicts of interest regarding the publication of this paper.

\section{Authors' Contributions}

Wuyang Huang and Zheng Yan contributed equally to this work.

\section{Acknowledgments}

This work was supported by the Natural Science Foundation Program of Jiangsu Province (BK20161376) and Jiangsu Agriculture Science and Technology Innovation Fund (CX(15)1026).

\section{Supplementary Materials}

Figure 1. Chromatographic separation and UV detection $(\lambda=520 \mathrm{~nm})$ of anthocyanins in blueberry anthocyanin extract. The following 14 peaks were identified: 1 , delphindin-3-galactoside; 2, delphindin-3-glucoside; 3, cyaniding-3-galactoside; 4, petunidin-3-galactoside; 5, cyaniding-3-glucoside; 6, cyaniding-3-arabinoside; 7, petunidin-3-glucoside; 8, peonidin-3-galactoside; 9, petunidin-3-arabinoside; 10, peonidin-3-glucosidea; 11, malvidin-3-galactoside; 12, malvidin-3-glucoside; 13, malvidin-3-arabinose; and 14, acylated anthocyanin. (Supplementary Materials)

\section{References}

[1] Y. Fan, Y. Qiao, J. Huang, and M. Tang, "Protective effects of Panax notoginseng Saponins against high glucose-induced oxidative injury in rat retinal capillary endothelial cells," Evidence-Based Complementary and Alternative Medicine, vol. 2016, Article ID 5326382, 9 pages, 2016.

[2] C.-f. Wang, J.-r. Yuan, D. Qin et al., "Protection of tauroursodeoxycholic acid on high glucose-induced human retinal microvascular endothelial cells dysfunction and streptozotocininduced diabetic retinopathy rats," Journal of Ethnopharmacology, vol. 185, pp. 162-170, 2016.

[3] M. Lorenzi and C. Gerhardinger, "Early cellular and molecular changes induced by diabetes in the retina," Diabetologia, vol. 44, no. 7, pp. 791-804, 2001.

[4] P. Zhang, Z. Zhang, and P. F. Kador, "Polyol effects on growth factors and MAPK signaling in rat retinal capillary cells," Journal of Ocular Pharmacology and Therapeutics, vol. 30, no. 1, pp. 4-11, 2014.

[5] D. S. London and B. Beezhold, "A phytochemical-rich diet may explain the absence of age-related decline in visual acuity of Amazonian hunter-gatherers in Ecuador," Nutrition Research, vol. 35, no. 2, pp. 107-117, 2015.
[6] D. Ghosh and T. Konishi, "Anthocyanins and anthocyaninrich extracts: role in diabetes and eye function," Asia Pacific Journal of Clinical Nutrition, vol. 16, no. 2, pp. 200-208, 2007.

[7] S. S. Paik, E. Jeong, S. W. Jung et al., "Anthocyanins from the seed coat of black soybean reduce retinal degeneration induced by N-methyl-N-nitrosourea," Experimental Eye Research, vol. 97, no. 1, pp. 55-62, 2012.

[8] S. H. Shim, J. M. Kim, C. Y. Choi, C. Y. Kim, and K. H. Park, "Ginkgo biloba extract and bilberry anthocyanins improve visual function in patients with normal tension glaucoma," Journal of Medicinal Food, vol. 15, no. 9, pp. 818-823, 2012.

[9] S. H. Lee, E. Jeong, S.-S. Paik et al., "Cyanidin-3-glucoside extracted from mulberry fruit can reduce $N$-methyl- $N$-nitrosourea-induced retinal degeneration in rats," Current Eye Research, vol. 39, no. 1, pp. 79-87, 2014.

[10] S. Nakamura, J. Tanaka, T. Imada, H. Shimoda, and K. Tsubota, "Delphinidin 3,5-O-diglucoside, a constituent of the maqui berry (Aristotelia chilensis) anthocyanin, restores tear secretion in a rat dry eye model," Journal of Functional Foods, vol. 10, pp. 346-354, 2014.

[11] W. Kalt, A. Hanneken, P. Milbury, and F. Tremblay, "Recent research on polyphenolics in vision and eye health," Journal of Agricultural and Food Chemistry, vol. 58, no. 7, pp. 40014007, 2010.

[12] Y. Wang, L. Zhao, F. Lu et al., "Retinoprotective effects of bilberry anthocyanins via antioxidant, anti-inflammatory, and anti-apoptotic mechanisms in a visible light-induced retinal degeneration model in pigmented rabbits," Molecules, vol. 20, no. 12, pp. 22395-22410, 2015.

[13] Y. Wang, D. Zhang, Y. X. Liu, D. Wang, J. Liu, and B. P. Ji, "The protective effects of berry-derived anthocyanins against visible light-induced damage in human retinal pigment epithelial cells," Journal of the Science of Food and Agriculture, vol. 95, no. 5, pp. 936-944, 2015.

[14] L. Bell, D. J. Lamport, L. T. Butler, and C. M. Williams, "A study of glycaemic effects following acute anthocyanin-rich blueberry supplementation in healthy young adults," Food \& Function, vol. 8, no. 9, pp. 3104-3110, 2017.

[15] S. A. Johnson, A. Figueroa, N. Navaei et al., "Daily blueberry consumption improves blood pressure and arterial stiffness in postmenopausal women with pre- and stage 1-hypertension: a randomized, double-blind, placebo-controlled clinical trial," Journal of the Academy of Nutrition and Dietetics, vol. 115, no. 3, pp. 369-377, 2015.

[16] Y. Liu, D. Zhang, J. Hu et al., "Visible light-induced lipid peroxidation of unsaturated fatty acids in the retina and the inhibitory effects of blueberry polyphenols," Journal of Agricultural and Food Chemistry, vol. 63, no. 42, pp. 9295-9305, 2015.

[17] J. Li, R. Deng, X. Hua et al., "Blueberry component pterostilbene protects corneal epithelial cells from inflammation via anti-oxidative pathway," Scientific Reports, vol. 6, no. 1, article 19408, 2016.

[18] Y. Liu, X. Song, Y. Han et al., "Identification of anthocyanin components of wild Chinese blueberries and amelioration of light-induced retinal damage in pigmented rabbit using whole berries," Journal of Agricultural and Food Chemistry, vol. 59, no. 1, pp. 356-363, 2011.

[19] C. Li, J. Feng, W.-Y. Huang, and X.-T. An, "Composition of polyphenols and antioxidant activity of rabbiteye blueberry (Vaccinium ashei) in Nanjing," Journal of Agricultural and Food Chemistry, vol. 61, no. 3, pp. 523-531, 2013. 
[20] E. C. Leal, C. A. Aveleira, Á. F. Castilho et al., "High glucose and oxidative/nitrosative stress conditions induce apoptosis in retinal endothelial cells by a caspase-independent pathway," Experimental Eye Research, vol. 88, no. 5, pp. 983-991, 2009.

[21] X. Zhu, K. Wang, K. Zhang et al., "Tetramethylpyrazine protects retinal capillary endothelial cells (TR-iBRB2) against IL-1 $\beta$-induced nitrative/oxidative stress," International Journal of Molecular Sciences, vol. 16, no. 9, pp. 21775-21790, 2015.

[22] T. Ago, T. Kitazono, H. Ooboshi et al., "Nox4 as the major catalytic component of an endothelial NAD(P)H oxidase," Circulation, vol. 109, no. 2, pp. 227-233, 2004.

[23] K. Schroder, M. Zhang, S. Benkhoff et al., "Nox4 is a protective reactive oxygen species generating vascular NADPH oxidase," Circulation Research, vol. 110, no. 9, pp. 1217-1225, 2012.

[24] F.-Y. Tang, F.-Y. Liu, and X.-W. Xie, "Association of angiotensin-converting enzyme and endothelial nitric oxide synthase gene polymorphisms with vascular disease in ESRD patients in a Chinese population," Molecular and Cellular Biochemistry, vol. 319, no. 1-2, pp. 33-39, 2008.

[25] A. Dursun, H. G. Durakbasi-Dursun, R. Dursun, S. Baris, and L. Akduman, "Angiotensin-converting enzyme gene and endothelial nitric oxide synthase gene polymorphisms in Behçet's disease with or without ocular involvement," Inflammation Research, vol. 58, no. 7, pp. 401-405, 2009.

[26] M. Barton, F. Cosentino, R. P. Brandes, P. Moreau, S. Shaw, and T. F. Luscher, "Anatomic heterogeneity of vascular aging: role of nitric oxide and endothelin," Hypertension, vol. 30, no. 4, pp. 817-824, 1997.

[27] D. F. Fitzpatrick, S. L. Hirschfield, T. Ricci, P. Jantzen, and R. G. Coffey, "Endothelium-dependent vasorelaxation caused by various plant extracts," Journal of Cardiovascular Pharmacology, vol. 26, no. 1, pp. 90-95, 1995.

[28] Y. Nakamura, H. Matsumoto, and K. Todoki, "Endotheliumdependent vasorelaxation induced by black currant concentrate in rat thoracic aorta," The Japanese Journal of Pharmacology, vol. 89, no. 1, pp. 29-35, 2002.

[29] P. J. Andrew and B. Mayer, "Enzymatic function of nitric oxide synthases," Cardiovascular Research, vol. 43, no. 3, pp. 521531, 1999.

[30] W. Y. Huang, X. N. Wang, C. Y. Li, Z. Yan, and W. M. Zhang, "Angiotensin I-converting enzyme inhibitory effects of malvidin and its glycosides from blueberry in endothelial cells," Basic \& Clinical Pharmacology \& Toxicology, vol. 119, p. 21, 2016.

[31] M. Clozel, G. A. Gray, V. Breu, B. M. Löffler, and R. Osterwalder, "The endothelin $\mathrm{ET}_{\mathrm{B}}$ receptor mediates both vasodilation and vasoconstriction in vivo," Biochemical and Biophysical Research Communications, vol. 186, no. 2, pp. 867-873, 1992.

[32] R. Gardlik and I. Fusekova, "Pharmacologic therapy for diabetic retinopathy," Seminars in Ophthalmology, vol. 30, no. 4, pp. 252-263, 2015.

[33] M. E. Cooper, D. Vranes, S. Youssef et al., "Increased renal expression of vascular endothelial growth factor (VEGF) and its receptor VEGFR-2 in experimental diabetes," Diabetes, vol. 48, no. 11, pp. 2229-2239, 1999.

[34] B. D. Manning and L. C. Cantley, "AKT/PKB signaling: navigating downstream," Cell, vol. 129, no. 7, pp. 1261-1274, 2007.

[35] A. K. Olsson, A. Dimberg, J. Kreuger, and L. Claesson-Welsh, "VEGF receptor signalling? in control of vascular function,"
Nature Reviews Molecular Cell Biology, vol. 7, no. 5, pp. 359371, 2006.

[36] L. Morbidelli, S. Donnini, and M. Ziche, "Role of nitric oxide in the modulation of angiogenesis," Current Pharmaceutical Design, vol. 9, no. 7, pp. 521-530, 2003.

[37] N. Jiang, X. L. Chen, H. W. Yang, and Y. R. Ma, "Effects of nuclear factor $\kappa \mathrm{B}$ expression on retinal neovascularization and apoptosis in a diabetic retinopathy rat model," International Journal of Ophthalmology, vol. 8, no. 3, pp. 448-452, 2015.

[38] L. Tornatore, A. K. Thotakura, J. Bennett, M. Moretti, and G. Franzoso, "The nuclear factor kappa B signaling pathway: integrating metabolism with inflammation," Trends in Cell Biology, vol. 22, no. 11, pp. 557-566, 2012.

[39] T. Khalfaoui, G. Lizard, and A. Ouertani-Meddeb, “Adhesion molecules (ICAM-1 and VCAM-1) and diabetic retinopathy in type 2 diabetes," Journal of Molecular Histology, vol. 39, no. 2, pp. 243-249, 2008.

[40] J. Paixão, T. C. P. Dinis, and L. M. Almeida, "Malvidin-3glucoside protects endothelial cells up-regulating endothelial NO synthase and inhibiting peroxynitrite-induced NF-kB activation," Chemico-Biological Interactions, vol. 199, no. 3, pp. 192-200, 2012.

[41] Y. Wang, Y. Huo, L. Zhao et al., "Cyanidin-3-glucoside and its phenolic acid metabolites attenuate visible light-induced retinal degeneration in vivo via activation of $\mathrm{Nrf} 2 / \mathrm{HO}-1$ pathway and NF- $\kappa \mathrm{B}$ suppression," Molecular Nutrition \& Food Research, vol. 60, no. 7, pp. 1564-1577, 2016. 


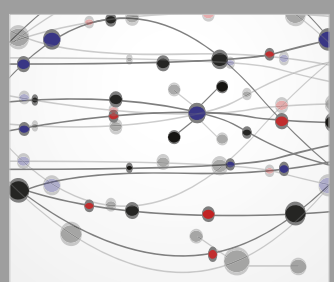

The Scientific World Journal
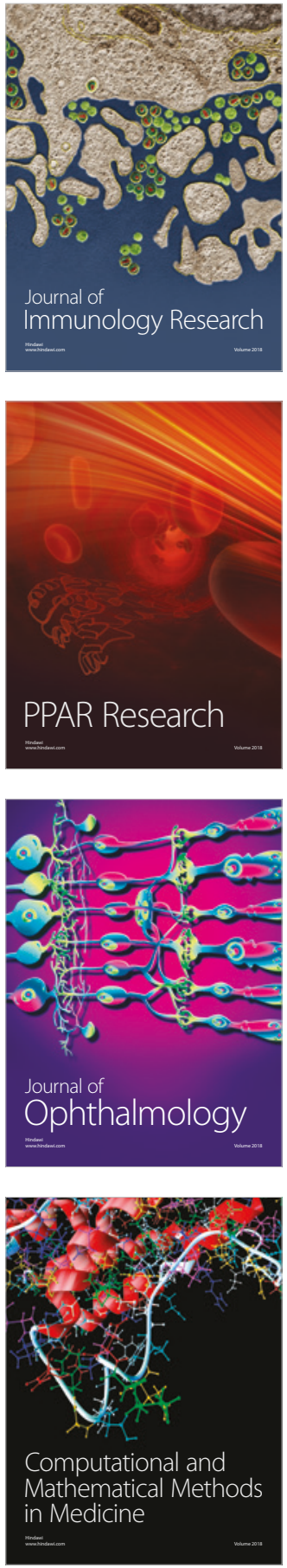

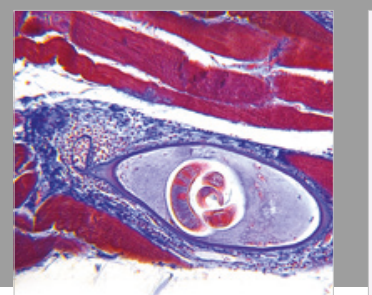

Gastroenterology Research and Practice

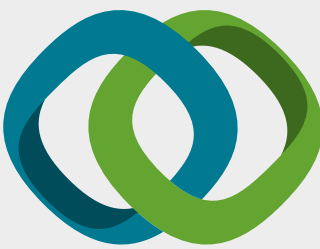

\section{Hindawi}

Submit your manuscripts at

www.hindawi.com
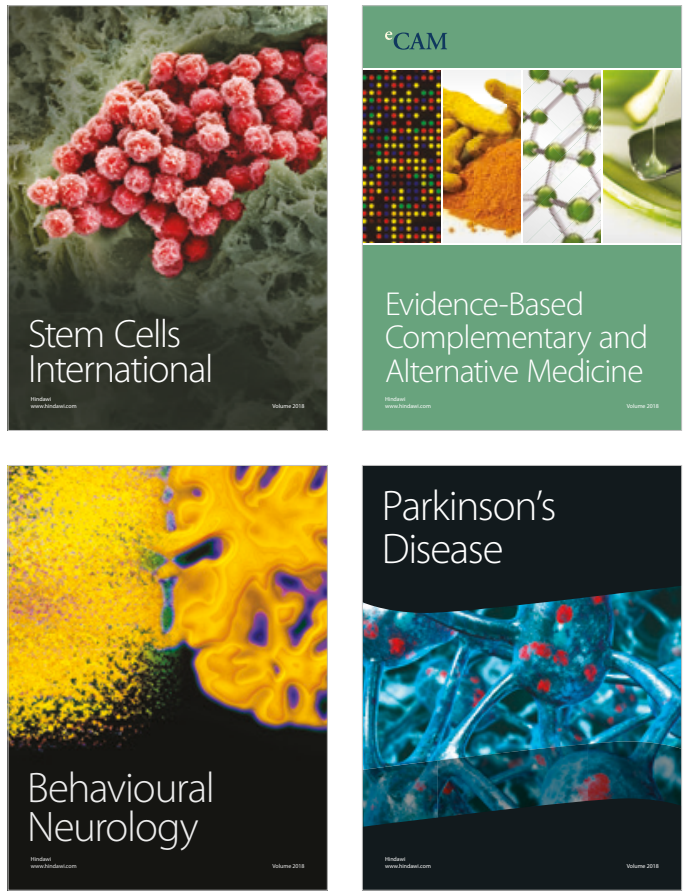

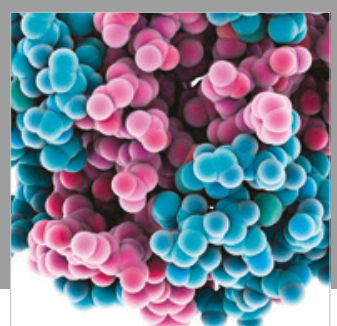

ournal of

Diabetes Research

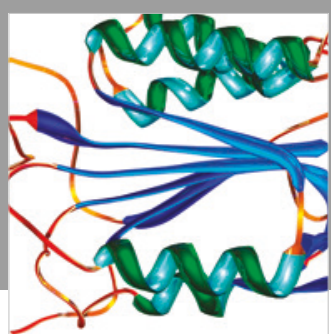

Disease Markers
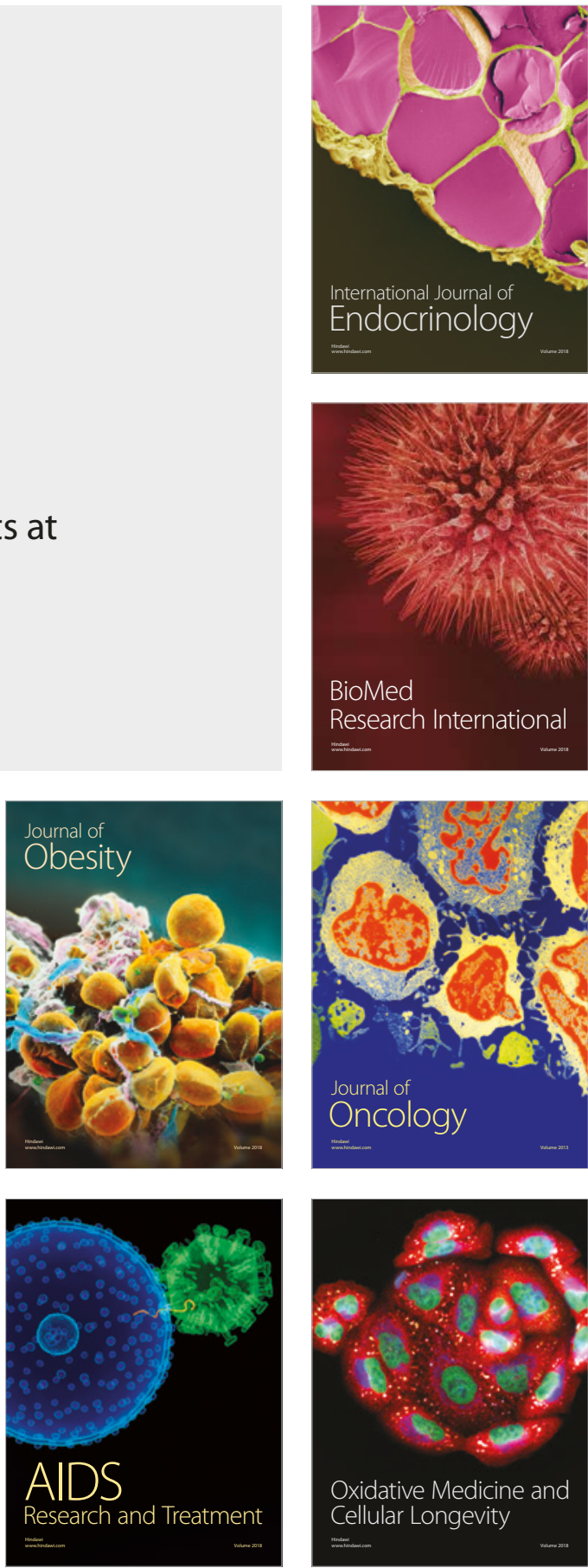